\title{
MacIntosh arthroplasty for the rheumatoid knee: 10-year follow up
}

\author{
B P WORDSWORTH, ${ }^{1}$ D T SHAKESPEARE, ${ }^{2}$ AND A G MOWAT
}

From the 'Department of Rheumatology and the ${ }^{2}$ Department of Orthopaedic Surgery, Nuffield Orthopaedic Centre, Oxford

SUMmaRY The results of 75 MacIntosh arthroplasties performed for rheumatoid arthritis of the knee in 63 patients were reviewed at least 10 years after surgery. Forty-two knees in 35 patients were available for assessment. Eleven arthroplasties had been revised to total knee replacemen $\vec{*}$ without difficulty because of pain or poor function. The remaining 31 knees in 25 patients gave good or excellent results in 22 cases, fair in eight, and poor in one. Seven patients could not be् traced, and 21 patients representing 26 knees had died. At least half these knees had given satisfactory results immediately before death judged by review of the case notes. The difficulty of comparing functional status with the preoperative state because of progressive multiarticula disease was highlighted. Although greater angular deformities preoperatively reduced the chancঙ of success in the medium term, late failure of the arthroplasty after five years was very rareळ Approximately two-thirds of all the arthroplasties performed gave satisfactory results at 10-yegr follow up or until the time of death.

The MacIntosh interposition arthroplasty was introduced in 1959, and we have used it in the surgical management of the rheumatoid knee since 1966. Blum et al. ${ }^{1}$ found impressive short-term results from 45 operations in this unit. Later Schorn et al. ${ }^{2}$ included the same group of patients within his review of 94 arthroplasties seen a mean of four years postoperatively. He found 49 good results, 23 fair, and 22 poor, of which 10 had been converted to total knee replacement (TKR). Of the 45 patients seen by both reviewers the results were unchanged in 28 $(62 \%)$, while four patients had improved, suggesting that results did not necessarily deteriorate with time. Examination of the long-term results was therefore desirable.

\section{Patients and methods}

All patients with definite rheumatoid arthritis (RA) who had undergone MacIntosh arthroplasty of one or both knees before 1972 in this hospital were identified. Surgery had been undertaken because of

Accepted for publication 24 April 1985.

Correspondence to Dr B P Wordsworth, Department of Rheumatology, Nuffield Orthopaedic Centre, Headington, Oxford OX3 7LD. the failure of conservative treatment to alleviat pain, to improve function, or to reduce deformity there being greater articular damage than could be treated by synovectomy alone. Those knees in whic? the original MacIntosh prosthesis remained were reviewed by a physician and a surgeon, neither of whom had been involved in the original surgery oi rheumatological care of the patients. Subjective anक्ष objective assessments of knee function and clinicat and radiographic measurements were made at fol $\not$. low up (Table 1) and compared with the pre- and postoperative assessments that had also been per? formed by independent observers. Functional abilie ties were graded on a four point scale, while joint movement and varus/valgus stability were measure重 with a goniometer.

Patellofemoral arthritis, varus/valgus angulation? prosthesis tilt and shift were measured from stan' ding radiographs. Patients were also asked to compare pain and overall function in the operate knee with those in the preoperative situation and asked whether in similar circumstances they would have the operation again. Problems in other joint\$ hindering the patient's progress and overall function were sought. The results were graded as excellent $\bar{\phi}$ good, fair, and poor according to symptomatip 
Table 1 Assessment of the efficacy of MacIntosh arthroplasty

\begin{tabular}{lll}
\hline Patient's assessment & Physician's assessment & Radiographic assessment \\
\hline Knee pain & Knee tenderness & Varus/valgus angle \\
Walking distance & Synovial proliferation & Prosthesis tilt \\
Rising from chair & Synovial effusion & Prosthesis shift \\
Climbing stairs & Range of flexion/extension & Patellofemoral arthritis \\
Use of walking aids & Varus/valgus rock & \\
Sleep & Anteroposterior glide & \\
\hline
\end{tabular}

relief and the patient's overall assessment of functional change (Table 2). Fixed criteria based upon the passive range of movement were not used to grade the results, since such ranges of movement may not be utilised during the performance of everyday activities. The results of surgery in those patients who had died were assessed from the case notes or by reference to the patient's general practitioner. In those cases where revision arthroplasty had been performed the timing and outcome of surgery were obtained from the case notes.

\section{Results}

Seventy-five arthroplasties had been performed in 63 patients during the relevant period (Table 3 ). Seven patients (seven knees) could not be traced. Twenty-one patients ( 26 knees) had died within 10 years of surgery, none as a result of surgical complications. Forty-two knees in 35 patients remained for assessment at 10 years or more, but 11 knees in ten patients had been revised to TKR

Table 2 Criteria for assessment of surgical results

\begin{tabular}{llll}
\hline Pain & Function & & \\
\cline { 2 - 4 } & $\begin{array}{l}\text { Normal or } \\
\text { much improved }\end{array}$ & $\begin{array}{l}\text { Same or } \\
\text { slightly better }\end{array}$ & Worse \\
\hline Absent & Excellent & Good & Fair \\
Improved & Good & Good & Fair \\
Same & Good & Fair & Poor \\
Worse & Fair & Poor & Poor \\
\hline
\end{tabular}

replacement because of pain or poor function. None of the prostheses had been infected, and revision arthroplasty was uncomplicated. Thirty-one knees (25 patients) in whom the original prosthesis remained were reviewed after a mean of 11.5 years (range 10-15 years). Pain and function compared with the pre-operative state are shown in Table 4. The range of flexion/extension was very similar before surgery $\left(10.5^{\circ}-97^{\circ}\right)$ and at follow up $\left(9.2^{\circ}-\right.$ $92^{\circ}$ ). Bizarre positions of the prosthesis were sometimes seen but were not necessarily inconsistent with a good or excellent result. The outcome of surgery assessed by the medical reviewers and patients is compared for those with uni- and bicompartmental procedures in Table 5 . The doctors tended to judge the result to be more successful than the patient. None of the results reached statistical significance, though the results tended to be slightly better when only one compartment had been operated upon. Comparison of functional abilities before and after surgery was difficult because 17 patients described pain and weakness in other joints holding them

Table 4 Knee function in 31 knees reviewed after at least 10 years compared with the preoperative state

\begin{tabular}{llcc}
\hline & Worse & Same & Better \\
\hline Pain in knee & 2 & 5 & 24 \\
Overall function & 2 & 3 & 26 \\
Walking distance & 7 & 8 & 16 \\
Rising from chair & 4 & 17 & 10 \\
Climbing stairs & 6 & 18 & 7 \\
Use of walking aids & 9 & 13 & 9
\end{tabular}

Table 3 Patients' details

\begin{tabular}{llllll}
\hline & Total & Follow up & Lost & Revised & Dead \\
\hline Number of patients & 63 & 25 & 7 & 10 & 21 \\
Number of knees & 75 & 31 & 7 & 11 & 26 \\
Sex (F:M) & $56: 7$ & $22: 3$ & $7: 0$ & $8: 2$ & $19: 2$ \\
Mean age (years) at operation (range) & $55(27-76)$ & $49(27-69)$ & $59(52-67)$ & $53(47-60)$ & $61(48-76)$ \\
Mean age (years) at follow up/death (range) & - & $60(41-79)$ & - & $-67(55-82)$ \\
Time of revision (years after operation) (range) & - & - & - & $5(1-12)$ & $3 \cdot 5(2-6)$ \\
& & & & & \\
\hline
\end{tabular}


Table 5 Overall physician's assessment and (patient's assessment)

\begin{tabular}{|c|c|c|c|c|}
\hline & Lateral compartmental & Medial compartmental & Bicompartmental & Total \\
\hline Excellent & $8(2)$ & $2(1)$ & $2(1)$ & $12(4)$ \\
\hline Good & $5(10)$ & $1(2)$ & $4(7)$ & $10(19)$ \\
\hline Fair & $2(4)$ & $0(0)$ & $6(3)$ & $8(7)$ \\
\hline Poor & $1(0)$ & $0(0)$ & $0(1)$ & $1(1)$ \\
\hline Total & $16(16)$ & $3(3)$ & $12(12)$ & $31(31)$ \\
\hline
\end{tabular}

back, markedly so in 12 cases. Only three patients decided they would not have the operation again in similar circumstances, one because of marked postoperative knee stiffness and two because of unrelieved knee pain.

In the 21 patients ( 26 knees) who had died review of the case notes suggested satisfactory function of the operated knee in 13 cases, unsatisfactory in four, uncertain in five, and four had been revised to TKR without difficulty. When these four revisions were included 15 MacIntosh arthroplasties had been revised to TKR a mean of 4.5 years (range 1-12 years) after the original surgery. Only one was revised after more than six years. Comparison of prosthesis position and the presence or otherwise of severe patellofemoral arthritis in patients requiring revision and in those who at follow up had good or excellent results showed no significant differences (Table 6). However, varus or valgus angulations of the knee tended to be greater preoperatively in those who required revision later $(\mathrm{p}<0 \cdot 1)$. Although angulation was corrected by surgery in both groups postoperatively, it tended to recur in those requiring revision later (Table 7).

Table 6 Comparison of prosthesis position in patients with good or excellent results and in those requiring revision

\begin{tabular}{|c|c|c|c|}
\hline & $\begin{array}{l}\text { Prosthesis tilt } \\
>20^{\circ}\end{array}$ & $\begin{array}{l}\text { Prosthesis shift } \\
>5 \mathrm{~mm}\end{array}$ & $\begin{array}{l}\text { Severe patello- } \\
\text { femoral arthritis }\end{array}$ \\
\hline 2 good results & s 7 & 12 & 11 \\
\hline 15 revisions & 3 & 4 & 7 \\
\hline
\end{tabular}

Table 7 Comparison of varus/valgus angle in patients with good or excellent results and in those requiring revision

\begin{tabular}{llll}
\hline & Preoperative Postoperative & $\begin{array}{l}\text { Ten years } \\
\text { later }\end{array}$ \\
\hline $\begin{array}{l}\text { Varus/valgus angle in } \\
22 \text { good results (deg) }\end{array}$ & 5.6 & 3.3 & 5.8 \\
$\begin{array}{l}\text { Varus/valgus angle in } \\
15 \text { revisions (deg) }\end{array}$ & 9.5 & 3.5 & 7.8 \\
\hline
\end{tabular}

\section{Discussion}

There is a dearth of information on the long-term results of prosthetic surgical management of the rheumatoid knee. A profusion of different types of TKR has appeared in recent years, but long-termresults remain uncertain. ${ }^{3}$ The MacIntosh arthro plasty is a less extensive surgical procedure requiring considerably less anaesthetic time than TKR, but ito has received a mixed press and is now relatively little used. ${ }^{4-6}$ The exact role of the prosthesis remains $Z$ conjectural, since in many instances it may be found in a non-weight-bearing site. At least some of the beneficial effects may be related to synovectomy which can still give acceptable results in moderately severely damaged joints. ${ }^{7}$ We found it difficult 900 predict those who would do well, though lar ger angular deformities and pronounced articular da age seemed to be adverse factors. Recurrence of angular deformity even after initial satisfactory correction was a relatively poor prognostic sign.

All attempts at long-term follow up suffer from the serious problem of progressive dwindling of the $\Rightarrow$ study population. Nevertheless, $42(56 \%)$ of our operated knees were available for study after at least 5 10 years. Thirty $(71 \%)$ of these had at leasto satisfactory results, and $22(52 \%)$ had good or excellent results. It was striking that late failure of the procedure was very unusual and only one of theo 15 patients undergoing revision arthroplasty had had his first operation more than six years previously. 0 We would therefore suggest that this procedure may still have a role to play in the surgical management? of the rheumatoid knee. Although the results in thes short term may not be quite as spectacular as the best reported with TKR, one may expect about twor thirds to have an acceptable result 10 years later or at the times of death. Good results in the medium 0 term can expect to be well maintained in the longw term. The procedure has the advantage of causingo relatively little surgical trauma and can easily beo converted at a later date to TKR if the result is unsatisfactory. This long-term follow up provides $a^{\infty}$ useful yardstick against which to judge the results of more recent designs of prosthetic management of the rheumatoid knee. 


\section{References}

1 Blum B. Mowat A G, Bentley G, Morris J R. Knee arthroplasty in patients with rheumatoid arthritis. Ann Rheum Dis 1974; 33: $1-11$.

2 Schorn D. Bentley G, Deane G, Mowat A G, MacIntosh arthroplasty in rheumatoid arthritis. Rheumatol Rehabil 1978; 17: $155-63$

3 Anonymous. Newer knowledge of total knee replacement [Editorial]. Clin Orthop 1979; 145: 2-3.

4 Anderson G B J. Jessop J. Freeman M R. Mason R M
Maclntosh arthroplasty in rheumatoid arthritis. Acta Orthop Scand 1974; 45: 245-59.

5 Hastings D E. Hewitson W A. Double hemi-arthroplasty of the knee in rheumatoid arthritis. J Bone Joint Surg 1973; 55B: $112-8$.

6 MacIntosh D L. Hunter G A. The use of the hemi-arthroplasty prosthesis for advanced osteoarthritis and rheumatoid arthritis of the knee. J Bone Joint Surg 1972; 54B: 244-55.

7 Geen S. Synovectomy and debridement of the knee in rheumatoid arthritis. J Bone Joint Surg 1969; 51A: 617-25.

\section{Book review}

Recent Advances in Systemic Lupus Erythematosus. By P H Lambert. L Perrin, S Izui. Pp. 359. £24.00. Academic Press: London. 1984.

This book is a report of a meeting on immunological and pathological aspects of systemic lupus erythematosus (SLE) which was held in Geneva in September 1983. It was also an opportunity to celebrate the 60th birthday of $\mathrm{Dr}$ Peter Miescher who has been a major contributor to the study of autoimmunity and particularly SLE. Many of the well known clinical and laboratory investigators concerned with SLE and autoimmunity are included in the 94 named participants. After an introduction on 'Current concepts of SLE', the sections are clustered as follows: two under 'Basic aspects of autoimmunity', four under 'SLE at the gene level', nine under 'Lymphocyte function and effector mechanisms', five under 'Pathology of SLE', and six under 'Present and future treatment in SLE'.

The format is that of a book of abstracts: judging from the differing type faces, style, and quality of editing the chapters have been assembled for printing without final editing. Some sections are notable for errors in typing, spelling, and accuracy of referencing, which might lead to scepticism as regards the accuracy of the contents. This is unfortunate, as the book contains a wealth of material for the clinical and laboratory investigator concerned with this area. One is also not sure how much was presented at the meeting but not included in the review: a contribution by Dr Aarden was apparently made at the meeting as judged by the reported discussion but is not included in the book.
Some of the sections are straightforward reviews, such as that on 'Antibodies to nonhistone nuclear antigens' by Tan and Wilson or the 'Haematological aspects of autoantibodies in SLE' by Gordon Smith and Harris. Some are restricted to detailed reporting of personal research, such as that by Drs Morel Maroger, Soderland, and Striker on the 'Characteristics of glomerular cells and their response to interferon:

The meeting was supported by the Sandoz Company, and the commercial interests of that organisation are apparent in one chapter on the use of cyclosporin A, and one on the use of intravenous gammaglobulin. That is not to say that there is any indication of a particular bias towards these products in this book: indeed they form a definitely coherent part of the section on treatment, which includes a useful and personal report by Dr Miescher and his colleagues on their own series of 85 patients.

In summary, this is a somewhat uneven presentation of what must have been a most stimulating and useful meeting for those participating. It would probably be most useful, therefore, as a record for those who actually participated. Nevertheless, it forms a valuable work of reference for both clinicians and laboratory scientists concerned with SLE.

Consultant Rheumatologist and

MICHAEL L SNAITH

Honorary Senior Lecturer

University College Hospital, London. 